\title{
Magnetic properties of the protostellar core IRAS 15398-3359*
}

\author{
E. Redaelli ${ }^{1}$, F. O. Alves ${ }^{1}$, F. P. Santos ${ }^{2}$, and P. Caselli ${ }^{1}$ \\ ${ }^{1}$ Centre for Astrochemical Studies, Max-Planck-Institut für extraterrestrische Physik, Gießenbachstraße 1, \\ 85749 Garching bei München, Germany \\ e-mail: eredaelli@mpe.mpg.de \\ ${ }^{2}$ Max-Planck-Institute for Astronomy, Königstuhl 17, 69117 Heidelberg, Germany
}

Received 9 July 2019 / Accepted 12 October 2019

\begin{abstract}
Context. Magnetic fields can significantly affect the star formation process. The theory of the magnetically driven collapse in a uniform field predicts that the contraction initially happens along the field lines. When the gravitational pull grows strong enough, the magnetic field lines pinch inwards, giving rise to a characteristic hourglass shape.

Aims. We investigate the magnetic field structure of a young Class 0 object, IRAS 15398-3359, embedded in the Lupus I cloud. Previous observations at large scales have suggested that this source evolved in an highly magnetised environment. This object thus appears to be an ideal candidate to study the magnetically driven core collapse in the low-mass regime.

Methods. We performed polarisation observations of IRAS $15398-3359$ at $214 \mu \mathrm{m}$ using the SOFIA telescope, thus tracing the linearly polarised thermal emission of cold dust.

Results. Our data unveil a significant bend of the magnetic field lines from the gravitational pull. The magnetic field appears ordered and aligned with the large-scale B-field of the cloud and with the outflow direction. We estimate a magnetic field strength of $B=78 \mu \mathrm{G}$, which is expected to be accurate within a factor of two. The measured mass-to-flux parameter is $\lambda=0.95$, indicating that the core is in a transcritical regime.
\end{abstract}

Key words. ISM: clouds - ISM: magnetic fields - stars: protostars - stars: formation - techniques: polarimetric ISM: individual objects: IRAS 15398-3359

\section{Introduction}

Magnetic fields $(B)$ are expected to play an important role in the star formation process, for instance providing a source of nonthermal pressure against the gravitational pull (see e.g. McKee \& Ostriker 2007). High-density molecular clouds are threaded by magnetic fields aligned perpendicularly to the main axes of the clouds (Planck Collaboration XXXV 2016), which is the expected configuration when $B$-fields regulate the star formation process more effectively than turbulence (Mouschovias \& Spitzer 1976; Nakamura \& Li 2008; Li et al. 2013). At smaller scales $(\approx 0.1-1 \mathrm{pc})$, the collapse of spherical cores in uniform fields have been widely investigated (Mouschovias 1991; Galli \& Shu 1993; Allen et al. 2003). Theory predicts the formation of a flattened structure (pseudo-disc) because the collapse happens preferentially along the field lines. Given that the interstellar gas is often slightly ionised, matter is expected to be coupled with the magnetic field lines at envelope scales. Therefore, magnetic lines pinch inwards as a result of the gravitational pull, exhibiting a characteristic hourglass shape. In low-mass star forming regions this feature has only been detected in $30 \%$ of young stellar objects in polarisation (9 sources out of 32; Hull \& Zhang 2019), suggesting that this is not a universal picture. Furthermore, out of these 9 detections only 2 show a clear hourglass shape, namely IRAS 4A (Girart et al. 2006) and L1448 (Kwon et al. 2018). Polarisation observations in the

\footnotetext{
* The Stokes maps are also available at the CDS via anonymous ftp to cdsarc.u-strasbg. fr (130.79.128.5) or via http://cdsarc. u-strasbg.fr/viz-bin/cat/J/A+A/631/A154
}

far-infrared (FIR) and sub-millimetre wavelength are an effective way to investigate the magnetic properties of clouds and cores at intermediate/high visual extinctions ( $\left.A_{\mathrm{V}} \gtrsim 10 \mathrm{mag}\right)$. In fact, asymmetric dust grains illuminated by an external radiation field are expected to develop a magnetic moment and thus to align with their minor axes parallel to the magnetic field direction, which is known as radiative torque alignment (RAT; Lazarian 2007). As a result, the background starlight at nearinfrared and optical frequency is absorbed and linearly polarised in the $B$-field direction. Similarly, since the dust grains emit preferentially in the direction of their major axes, dust thermal emission is linearly polarised perpendicularly to the local $B$-field. Cold dust $(T \approx 10-30 \mathrm{~K})$ emission happens typically at FIR or sub-millimetre wavelengths.

IRAS 15398-3359 (hereafter IRAS 15398) is a low-mass Class 0 protostar (Andre et al. 2000) located in the Lupus I molecular cloud at a distance of $156 \mathrm{pc}$ (Dzib et al. 2018). The protostellar mass, estimated from modelling of the envelope rotation, is subsolar $\left(M_{*}<0.1 M_{\odot}\right.$; Oya et al. 2014; Yen et al. 2017). Lupus I is the least evolved cloud of the Lupus complex (Rygl et al. 2013), and optical polarisation observations have shown that it is threaded by a very ordered magnetic field, perpendicular to its whole filamentary extension (Franco \& Alves 2015). IRAS 15398 has a luminosity of $1.8 L_{\odot}$ and an envelope mass of $1.2 M_{\odot}$. It powers a bipolar outflow detected in $\mathrm{CO}$ lines both with single dish and interferometric observations (Tachihara et al. 1996; Bjerkeli et al. 2016).

Using the Stratospheric Observatory for Infrared Spectroscopy (SOFIA) telescope, we observed the polarised dust thermal emission arising from the core and the filamentary 
structure in which IRAS 15398 is embedded. For the first time, we can compare the large-scale field with the small-scale field in a Class 0 source. In this paper, we report our findings, which unveil an ordered magnetic field, which hints of pinching of the field lines resulting from the gravitational pull. Our results suggest that IRAS 15398 evolved in a highly magnetised environment and experienced a magnetically driven collapse.

\section{Observations}

\subsection{Polarimetric data}

The polarimetry observations were carried out with the SOFIA telescope, using the High-resolution Airborne Wideband Camera Plus (HAWC+) instrument (Harper et al. 2018) in summer 2018. We used the band $E$, which has a nominal wavelength of $214 \mu \mathrm{m}(1.4 \mathrm{THz})$. At this frequency, the SOFIA beam full width half maximum (FWHM) is $F W H M \approx 19^{\prime \prime}$ (corresponding to $\approx 0.01 \mathrm{pc}$ at the distance of the source) and the field of view is $5.0^{\prime} \times 6.3^{\prime}$. The data were processed with the standard pipeline (HAWC DRP, version 1.3.0), and we used the fluxcalibrated Level 4 data products (already flux calibrated and mosaicked). The total integration time was $\approx 1 \mathrm{~h} 15 \mathrm{~min}$, and the sensitivity achieved in the Stokes $I$ is $65 \mathrm{mJy}^{\text {beam }}{ }^{-1}$. In order to increase the signal-to-noise ratio $(\mathrm{S} / \mathrm{N})$ of the data and to have significant detections over a large percentage of the source, we smoothed the Stokes parameter $I, Q$, and $U$ to $42^{\prime \prime}$ resolution $(\approx 0.03 \mathrm{pc})$, before producing maps of polarisation intensity and position angle (see Sect. 3). The final mean sensitivity is $\mathrm{rms}=48 \mathrm{mJy}_{\text {beam }}^{-1}$ (Stokes $\left.I\right), 77 \mathrm{mJy} \mathrm{beam}^{-1}$ (Stokes $\left.Q\right)$, and $77 \mathrm{mJy}^{-1}$ beam ${ }^{-1}$ (Stokes $U$ ). Since we found no indication of spatially correlated noise, the smoothing technique as a tool to increase $\mathrm{S} / \mathrm{N}$ is justified.

\section{2. $\mathrm{H}_{2}$ column density maps}

To obtain the gas column density $N\left(\mathrm{H}_{2}\right)$ and the visual extinction $A_{\mathrm{V}}$ maps of the source, we used the archive data of the Gould Belt Survey, performed with the Herschel space telescope (André et al. 2010). These data provide directly the $\mathrm{H}_{2}$ column density map of Lupus I, obtained with a spectral fitting of the dust emission at five wavelengths $(70,160,250$, 350 , and $500 \mu \mathrm{m})$. This map has a resolution of $\approx 38^{\prime \prime}$, which allows for a fair comparison with the SOFIA smoothed data. We derived the visual extinction map using the standard relation $A_{\mathrm{V}} / \mathrm{mag}=1.06 \times 10^{-21} \times N\left(\mathrm{H}_{2}\right) / \mathrm{cm}^{-2}$, (Bohlin et al. 1978) .

\section{Results}

Appendix A reports the maps of the three Stokes parameters $I$, $Q$, and $U$. From these, we derive the polarised flux $\left(I_{\mathrm{P}}\right)$, polarised fraction $(P)$, and polarisation angle (PA), following the standard equations:

$I_{\mathrm{P}}=\sqrt{Q^{2}+U^{2}-\sigma_{I_{\mathrm{P}}}^{2}}$

$P=\frac{I_{\mathrm{P}}}{I}$,

$\mathrm{PA}=\frac{1}{2} \arctan \left(\frac{U}{Q}\right)$.

In order to remove the bias of the polarised intensity, in Eq. (1) we removed the contribution of the flux uncertainty $\left(\sigma_{I_{\mathrm{P}}}\right)$. This procedure is necessary since $I_{\mathrm{P}}$ is biased to positive values,
Table 1. Peak and mean values of the polarised flux, polarised fraction, and position angle.

\begin{tabular}{ccc}
\hline \hline Parameter & Peak & Mean $^{(a)}$ \\
\hline$I_{\mathrm{P}}\left(\mathrm{Jy} \mathrm{beam}^{-1}\right)$ & $0.53 \pm 0.07$ & $0.28 \pm 0.07$ \\
$P(\%)$ & $28 \pm 8$ & $6.6 \pm 1.8$ \\
$P A\left(^{\circ}\right)$ & - & $45 \pm 7$ \\
\hline
\end{tabular}

Notes. ${ }^{(a)}$ The mean values are computed over positions satisfying $\sigma_{\mathrm{P}} / P>3.0$ and $P<30 \%$.

while the Stokes $U$ and $Q$ parameters can be positive or negative (Vaillancourt 2006). To avoid oversampling, after smoothing the data we re-gridded the maps to a pixel size of $9.1^{\prime \prime}(\approx 4$ pixels per smoothed beam). Table 1 summarises the peak and mean values for the derived parameters. Figure 1 shows the Stokes $I$ emission, with polarisation vectors already rotated by $90^{\circ}$ to indicate the plane-of-sky component of the magnetic field. We masked data points with a $\mathrm{S} / \mathrm{N}$ in a polarisation fraction lower than 3.

\section{Analysis and discussion}

\subsection{Magnetic field direction}

Figure 1 shows the magnetic field direction traced by the SOFIA data. The field at sub-parsec scale is aligned with the large (parsec) scale field observed in starlight optical polarisation. The histograms of the two position angle distributions are presented in Fig. 2. The mean values of the two distributions differ by $\approx 5^{\circ}$, which is less than the mean uncertainty on the SOFIA position angles $\left(\left\langle\epsilon_{\mathrm{PA}}\right\rangle=7^{\circ}\right)$. Since the two datasets are sensitive to two different regimes, i.e. the optical data trace large cloud scale, whilst the SOFIA data are sensitive to the core scales, this means that the uniform magnetic field of the cloud has been inherited by the core and that the gravitational collapse was magnetically driven.

In Fig. 1 we also show that the outflow direction $\left(\mathrm{PA}=35^{\circ}\right.$, Bjerkeli et al. 2016) lies almost parallel to the magnetic field, which has a mean direction of $\langle\mathrm{PA}\rangle=(45 \pm 7)^{\circ}$. This behaviour is consistent with the prediction of the theory of the magnetically driven collapse, according to which a strong magnetic field can efficiently remove the excess of angular momentum from rotating cores, thus aligning the rotation axis with the magnetic axis (see e.g. Li et al. 2014, and references therein).

Another prediction of the theory is the hourglass shape of the magnetic field lines. In IRAS 15398 this is not clearly visible, even though we detect a bending of the field lines which may hint to a partial hourglass shape. On the side of the core facing south-east, the field lines pinch inwards towards the centre of the object. The mean position angle north-east of the source is $\mathrm{PA}=(55 \pm 7)^{\circ}$, whilst $\mathrm{PA}=(36 \pm 7)^{\circ}$ is derived south-west of the source. The change in angle is thus $19^{\circ}$, significantly larger than the uncertainty. We do not detect the north-west side of the hourglass, possibly for two reasons. First of all, on that side we lack the sensitivity to detect a bend in the field lines, since the polarised flux is less prominent. This can be due to the presence of the filamentary structure that extends towards the west. This filament provides more shielding from the external radiation field with respect to the other side of the core, which is more exposed. Since this radiation is responsible for the grain alignment, according to the RAT theory, grains are less efficiently aligned on the north-west side of IRAS 15398, which results in a lower polarised flux. Moreover, the presence of the filament 


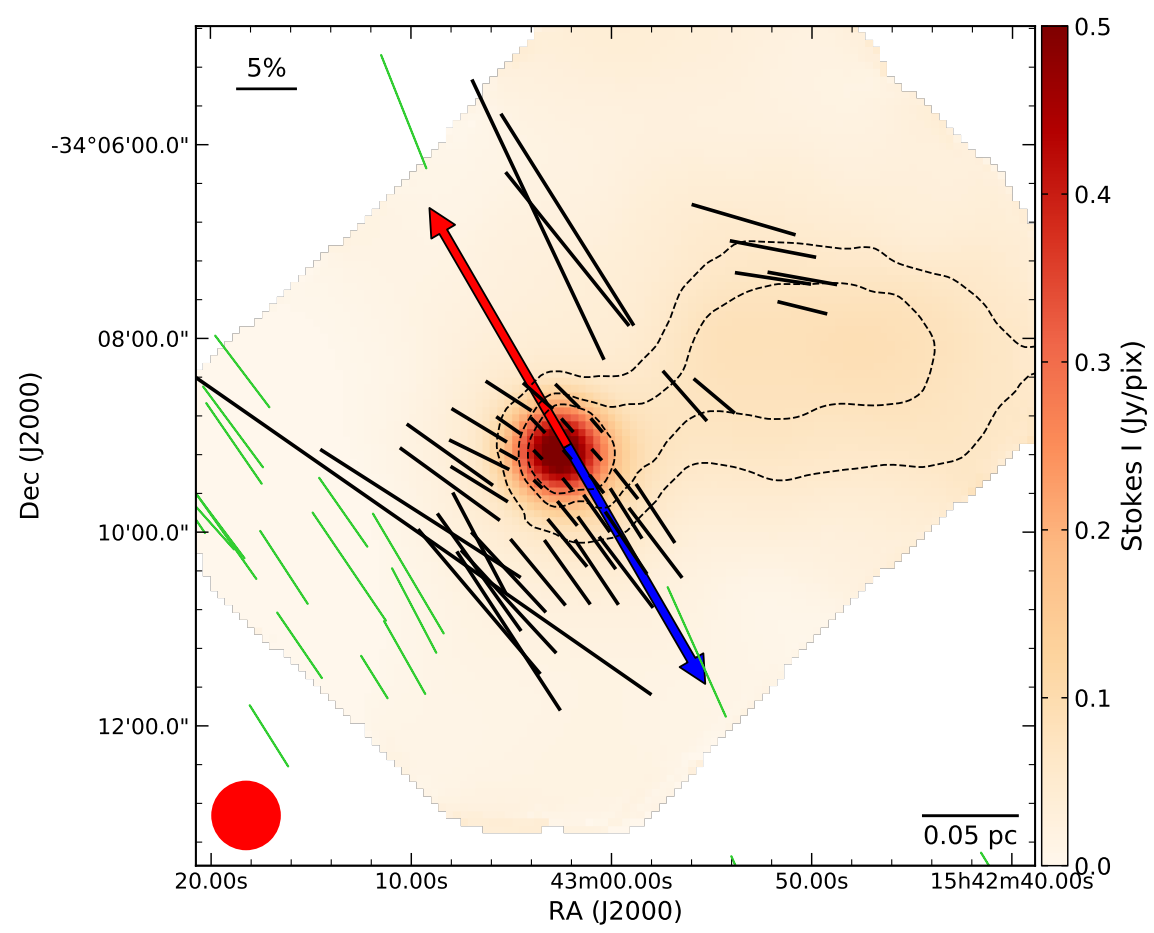

Fig. 1. Colour-scale map of the Stokes $I$ flux observed by SOFIA. The dashed contours represent $N\left(\mathrm{H}_{2}\right)$ column density as derived from Herschel data (levels $=[1.0,1.5,2.0] \times 10^{22} \mathrm{~cm}^{-2}$ ). The black vectors show the polarisation angles, tilted by $90^{\circ}$ to trace the magnetic field direction, and their length is proportional to the polarisation fraction (scale bar in the top left corner). To show statistically independent data, we plot only two vectors per smoothed beam. The smoothed beam size is shown in the bottom left corner. In green, we show the polarisation vectors from optical observations from Franco \& Alves (2015). The red and blue arrows indicate the direction of the outflow.

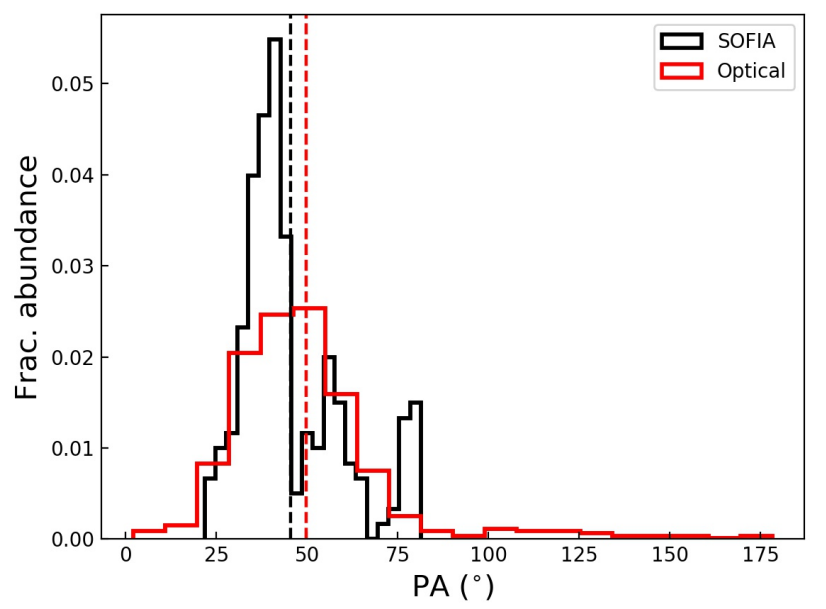

Fig. 2. Fractional abundance distribution of the magnetic field direction angles as traced by the THz data (black) and optical data (red). The distributions mean values, shown with vertical dashed lines, are $45^{\circ}$ (SOFIA) and $50^{\circ}$ (optical). The SOFIA data present a three peaked distribution, with peaks being at $\approx 40^{\circ}, \approx 55^{\circ}$, and $\approx 77^{\circ}$. These correspond to vectors coming from the two halves of the presumed hourglass shape and filament, respectively.

can affect the detected morphology of the field lines in a second way. The presence of the extended dust emission in the west direction could have played a role in disturbing the spherical collapse of the core. This scenario is supported by the few detections along the filament, which reveal a significant twist in the $B$ direction $\left(\mathrm{PA} \approx 69^{\circ}\right)$. This suggests that in this portion of the source the magnetic field morphology has been perturbed, likely by accretion motions towards the central object.

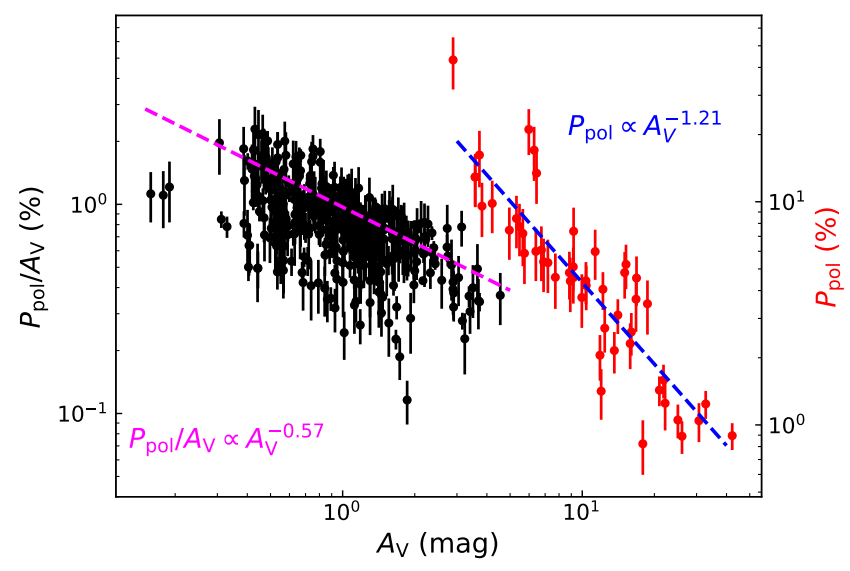

Fig. 3. Scatter plots of the polarisation efficiency as a function of the visual extinction in magnitudes for the optical data (in black) and for the FIR data (in red, only one point every other is shown), in logarithmic scale. The best fit to each dataset, as described in the main text, is shown with the pink and blue dashed curve, respectively. The best-fit equations are also shown in the bottom left and top right corners.

\subsection{Depolarisation at high column densities}

Figure 1 shows that the emission is less polarised as the column density gets higher, towards the centre of the core. This effect has been widely observed in the literature (Matthews et al. 2009; Alves et al. 2014; Jones et al. 2015, 2016). In Fig. 3 we show the scatter plot of the polarisation fraction as a function of the visual extinction. We also report the polarisation efficiency for the optical data, which is defined as the polarised fraction normalised by the visual extinction (see the Introduction in Santos et al. 2017). In this way, we correctly take into account the attenuation 
of the background stellar light. We use the data from Franco \& Alves (2015), limiting ourselves to the galactic latitude range $16.2^{\circ}<b<17.0^{\circ}$ (the "Middle" region in Franco \& Alves 2015).

There are a number of possible explanations for the depolarisation at high column densities. In general, if the alignment is dominated by radiative torque due to the interstellar radiation field, at higher visual extinction less and less radiation penetrates the core and dust grains are less aligned. Moreover, the alignment efficiency is highly sensitive to the dust grain population (Bethell et al. 2007; Pelkonen et al. 2009; Brauer et al. 2016). In particular, Brauer et al. (2016) showed that polarisation can decrease by up to $10 \%$ when grains are submillimetre or millimetre in size. Grain growth is expected in Class 0 objects, as shown by Jørgensen et al. (2007), Kwon et al. (2009) and Chiang et al. (2012). Finally, geometrical smearing is possible. Close to the central object, a highly perturbed magnetic field is likely to be present because of the dominant effect of gravity at the small scales which are unresolved by the SOFIA data. Higher angular resolution observations are needed to disentangle these scenarios.

We fitted a linear relation in the $\log -\log$ space for the polarisation efficiency versus visual extinction in each dataset, deriving the slope of the relation $P_{\text {eff }} \propto A_{\mathrm{V}}^{-\alpha}$ (Fig. 3). For the optical data, tracing the cloud scales, we find $\alpha=0.57 \pm 0.07$, which is consistent with modelling of RAT alignment in molecular clouds (see e.g. Whittet et al. 2008, who also report the measurements in Taurus and Ophiucus clouds). For the FIR data, we find a steeper slope ( $\alpha=1.21 \pm 0.12)$, implying a strong depolarisation for $10 \lesssim A_{\mathrm{V}}(\mathrm{mag}) \lesssim 50$. Values of $\alpha \approx 1$ are often found in literature for prestellar and young Class 0 objects (Alves et al. 2014; Jones et al. 2015, 2016). The different slopes exhibited by the optical and FIR data suggest that the two datasets are probing two different regimes in the grain alignment. This in turn can be related to two different grain populations, in which the larger grains in the core are less efficiently aligned to the $B$-field than those in the cloud (see e.g. Sect. 3 in Andersson et al. 2015), or it can be due to a less efficient RAT, in which the grains traced by SOFIA are more shielded from the interstellar radiation field with respect to those traced by the optical data. Another possibility is a more entangled magnetic field lines, as mentioned before. According to recent results, a slope of $\alpha \approx 1$ can be a consequence of biases introduced by the $\mathrm{S} / \mathrm{N}$ cut (Wang et al. 2019). To exclude this possibility, we fitted the linear relation lowering the threshold to $S / N>0.5$, obtaining $\alpha=1.26 \pm 0.09$, in agreement the previous result, thus suggesting that no significant bias is introduced by the data selection.

\subsection{Angular dispersion function and field strength}

The angular dispersion function (ADF) method, introduced by Hildebrand et al. (2009) and Houde et al. (2009), involves the computation of the autocorrelation function of the position angles. It allows us to derive quantitative information on the B-field without depending on an assumption concerning the field morphology. The method assumes that the magnetic field is composed by an ordered component $\left(B_{0}\right)$, slowly changing spatially, and a turbulent or random component $\left(B_{\mathrm{t}}\right)$, which is characterised by a coherent length scale $\delta$. We now consider the autocorrelation function of the position angles $\Delta \Phi$, i.e. the difference in angle of every pair of vectors separated by the distance $l$ as follows:

$\left\langle\Delta \Phi^{2}(l)\right\rangle=\frac{1}{N} \sum_{i=1}^{N}[\Phi(x)-\Phi(x+l)]^{2}$.
Hildebrand et al. (2009) showed that on scales $d$ such that $\delta<d \ll \Delta$, where $\Delta$ is the physical scale of the analysed source, the structure function has the form

$\left\langle\Delta \Phi^{2}(l)\right\rangle=b^{2}+a^{2} l^{2}+\sigma_{\mathrm{M}}^{2}$,

where $\sigma_{\mathrm{M}}$ is the uncertainty on the measurements. The parameter $a$ is linked to the large-scale variations of the $B$-field, while $b$ is related to the ratio of the turbulent and uniform magnetic field components via $B_{\mathrm{t}} / B_{0}=b / \sqrt{2-b^{2}}$.

Houde et al. (2009) further expanded the analysis, in particular including the effects introduced by the telescope beam size. In case of a Gaussian beam with standard deviation $W$, they found the following expression:

$$
\begin{aligned}
\left\langle\Delta \Phi^{2}(l)\right\rangle= & 2 \sqrt{2 \pi}\left(\frac{B_{\mathrm{t}}}{B_{0}}\right)^{2} \frac{\delta^{3}}{\left(\delta^{2}+2 W^{2}\right) \Delta^{\prime}} \\
& \times\left[1-\exp \left(-\frac{l^{2}}{2\left(\delta^{2}+2 W^{2}\right)}\right)\right]+m^{2} l^{2},
\end{aligned}
$$

where $\Delta^{\prime}$ is the cloud effective thickness and $m$ is a parameter related to the large-scale structure of the magnetic field, which does not involve turbulence.

In order to fit Eq. (6) to derive the $B_{\mathrm{t}} / B_{0}$ parameter, we computed $\Delta \Phi^{2}$ for all the available pairs of points with $0^{\prime \prime}<l<180^{\prime \prime}$, divided in 9 bins $20^{\prime \prime}$ wide. The choice of binning is determined by the angular resolution of the observations, as we did not want to oversample the beam size, and by the map size; each bin should contain a fairly constant number of points. We computed the uncertainty $\sigma_{\mathrm{M}}$ in each bin propagating the uncertainties on the position angles, assumed to be uncorrelated. They are usually within $3-5 \%$. The FWHM of SOFIA smoothed beam (42") corresponds to a standard deviation of $W=18^{\prime \prime}=13 \mathrm{mpc}$.

Because of the limited number of data points available for the fit, we fixed the turbulence correlation scale on $\delta=20 \mathrm{mpc}$. This value is in good agreement with previous estimation of this quantity in other star forming regions: for instance Houde et al. (2009) found $\delta=16 \mathrm{mpc}$ in OMC-1, Frau et al. (2014) derived $\delta=13-33 \mathrm{mpc}$ in the high-mass star forming region NGC 7538, and Coudé et al. (2019) reported $\delta=7 \mathrm{mpc}$ in Barnard 1. Furthermore, we assumed the cloud thickness $\Delta^{\prime}$ to be equal to the source effective radius $\left(r_{\text {eff }}\right)$, defined as the radius of a circular region of identical surface area. To compute the latter, we considered only positions with Stokes $I>0.05 \mathrm{Jy} \mathrm{pix}^{-1}$. This threshold roughly corresponds to the lowest contour in $N\left(\mathrm{H}_{2}\right)$ in Fig. 1 $\left(1 \times 10^{22} \mathrm{~cm}^{-2}\right)$, and it is the first closed contour that comprises both the central core and filamentary structure towards west. We obtained $r_{\text {eff }}=0.1 \mathrm{pc}$, a result which is also in agreement with the typical size of cores and filamentary structures in molecular clouds (see e.g. Arzoumanian et al. 2011; André et al. 2014). In Sect. 4.4 we explored the effects of these assumptions on the results.

In Fig. 4 we show the resulting data points and their uncertainties. We also show the best-fit solution found for Eq. (6). The obtained best-fit parameters are reported in Table 2. We emphasise that while figures and tables are expressed in degree and arcsecond units, the actual fit is performed in radiant and parsec units.

To estimate the strength of the magnetic field, we applied a modified version of the method proposed by Chandrasekhar \& Fermi (1953, hereafter CF). The CF approach assumes the equipartition of kinetic and perturbed magnetic field energy to link the magnetic field strength on the plane of sky $\left(B_{\mathrm{pos}}\right)$ to the velocity dispersion of the gas $\left(\sigma_{\mathrm{V}}\right)$ and to the dispersion of the 


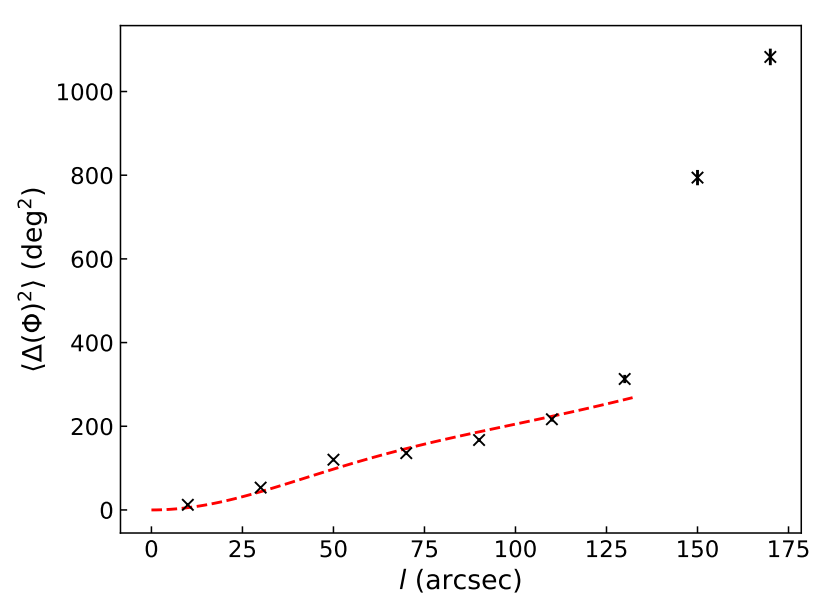

Fig. 4. Angular dispersion function of the SOFIA data with respect to the distance parameter $l$, computed as described in Sect. 4.3. The measurement uncertainties $\sigma_{\mathrm{M}}$ are shown as error bars, and for low values of $l$ they are too small to be seen. The best fit to the data points, following Eq. (6), is shown with a dashed curve.

Table 2. Summary of the main parameters concerning the magnetic field, derived as described in Sect. 4.3.

\begin{tabular}{cccc}
\hline \hline$B_{\mathrm{t}} / B_{0}$ & $m\left(\mathrm{deg}^{2} / \operatorname{arcsec}^{2}\right)$ & $B_{\text {pos }}(\mu \mathrm{G})$ & $\lambda$ \\
\hline $0.267 \pm 0.007$ & $170 \pm 50$ & 78 & 0.95 \\
\hline
\end{tabular}

polarisation angle $(\delta \phi)$, which in turn corresponds to the turbulent ratio $\left(\delta \phi \equiv B_{\mathrm{t}} / B_{0}\right)$. Further development of the theory led to the equation

$B_{\mathrm{pos}}=\sqrt{4 \pi \mu m_{\mathrm{H}} n_{\mathrm{H}_{2}}} \frac{\sigma_{\mathrm{V}}}{\delta \phi}$,

which is valid in the assumption of a small polarisation angle $\left(\delta \phi<25^{\circ}\right.$; Ostriker et al. 2001; Padoan et al. 2001; Crutcher et al. 2004). In Eq. (7), $\mu$ is the gas mean molecular weight per hydrogen molecule, assumed to be $\mu=2.8$ (Kauffmann et al. 2008), $m_{\mathrm{H}}$ the hydrogen mass $\left(m_{\mathrm{H}}=1.6710^{-24} \mathrm{~g}\right)$, and $n_{\mathrm{H}_{2}}$ is the gas volume density.

To compute $B_{\text {pos }}$, we needed to determine the quantities $n_{\mathrm{H}_{2}}$ and $\sigma_{\mathrm{V}}$. For the gas volume density, we consider the $\mathrm{H}_{2}$ column density map, integrated over the region used to derive the effective radius, obtaining the total number of $\mathrm{H}_{2}$ molecules $H_{\text {tot }}$ in the considered region. Assuming a uniform distribution of the gas, we could thus derive

$n_{\mathrm{H}_{2}}=\frac{3}{4 \pi} \frac{H_{\mathrm{tot}}}{r_{\mathrm{eff}}^{3}}=2.6 \times 10^{4} \mathrm{~cm}^{-3}$.

For the gas velocity dispersion, we used the results of Benedettini et al. (2012), who observed several molecular tracers in Lupus I with the MOPRA telescope, which at $3 \mathrm{~mm}$ has a beam size similar to the one of our data. At the position of IRAS 15398, these authors reported FWHM values in the range $0.31-0.72 \mathrm{~km} \mathrm{~s}^{-1}$. We adopted the mean of their results (excluding the CS (2-1) line, which is most likely optically thick), $F W H M=0.41 \mathrm{~km} \mathrm{~s}^{-1}$, corresponding to a velocity dispersion $\sigma_{\mathrm{V}}=0.17 \mathrm{~km} \mathrm{~s}^{-1}$. Inserting these values in Eq. (7), we obtained $B_{\text {pos }}=78 \mu \mathrm{G}$. Owing to the strong assumptions of the method, such as the core uniform density, we expected this value to be accurate within a factor of two. Furthermore, we want to highlight that it is intrinsically a lower limit, since it neglects the line-of-sight component of the field.

A fundamental parameter is the mass-to-flux ratio $(M / \Phi)$, which is determined by the ratio of the gravitational to magnetic energy. This ratio provides the dynamical state of the core (equilibrium or collapse). In particular, it is interesting to compute its observed value with respect to the critical value, defined by the parameter $\lambda$ (Crutcher et al. 2004), i.e.

$\lambda=(M / \Phi)_{\text {obs }} /(M / \Phi)_{\text {crit }}=7.6 \times 10^{-21}\left(\frac{N\left(\mathrm{H}_{2}\right)}{\mathrm{cm}^{-2}}\right)\left(\frac{B}{\mu \mathrm{G}}\right)^{-1}$,

where $N\left(\mathrm{H}_{2}\right)$ is the mean column density of the source, computed in the region over which $\mathrm{B}$ is measured. Focussing on the same region used to derive $n_{\mathrm{H}_{2}}$, we obtained $N\left(\mathrm{H}_{2}\right)=9.8 \times$ $10^{21} \mathrm{~cm}^{-2}$, and therefore $\lambda=0.95$. Our derived value of $\lambda$ suggests that the core is in transitional state between being subcritical and supercritical.

Our analysis of the mass-to-flux ratio considers only the gas mass of the source, and neglects the protostellar mass. However, the total mass within the contour Stokes $I>0.05 \mathrm{Jy} \mathrm{pix}^{-1}$ is $\approx 6 M_{\odot}$, which is significantly higher than the protostellar mass (the upper limit is $M_{*}<0.1 M_{\odot}$, Yen et al. 2017). This approximation therefore causes an error of less than $2 \%$, i.e. well below our uncertainties.

\subsection{Influence of the assumed parameters}

The analysis described above depends on the assumption of several key parameters. We explore in this section how the results are affected by changing the assumed values. The first assumption regards the turbulence correlation scale, $\delta$. The literature results seem to suggest that $\delta$ is lower for low-mass star forming regions with respect to high-mass ones: both Liu et al. (2019) and Coudé et al. (2019) found $\delta<10 \mathrm{mpc}$ in low-mass cores. We therefore tested other three values $(5,10,15 \mathrm{mpc})$, obtaining $B_{0}=16,40,62 \mu \mathrm{G}$, respectively. The magnetic field strength hence increases with increasing $\delta$. The turbulent-touniform component ratio is lower than one in all cases but the extreme one $(\delta=5 \mathrm{mpc})$, for which is marginally higher than one $\left(B_{\mathrm{t}} / B_{0}=1.3\right)$. On the other hand, the mass-to-flux ratio for these values is $\lambda>1.0$, pushing the state of the core more towards the supercritical regime.

A second assumption that is made regards the cloud effective thickness $\left(\Delta^{\prime}\right)$, which may not necessary correspond to the real thickness $\Delta$. In particular, according to Houde et al. (2009), $\Delta^{\prime} \leq$ $\Delta$, where the equality holds only if the large-scale magnetic field is completely uniform. We therefore repeated the analysis using $\Delta^{\prime}=r_{\text {eff }} / 2$. The results vary of less than $30 \%$, which is below our uncertainties.

The magnetic field strength can also be calculated directly with the modified Chandrasekhar-Fermi method (Eq. (7)), computing directly the standard deviation of the position angles $(\delta \phi)$, hence avoiding the assumptions made in the ADF method. In this case, Eq. (7) must be corrected for a numerical factor $(Q)$ to take into account that its original version tends to overestimate the magnetic field (Ostriker et al. 2001; Crutcher et al. 2004). A usually adopted value is $Q=0.5$. From the SOFIA data, we measured $\delta \phi_{\mathrm{obs}}=14^{\circ}$. This value is however biased upward by the observational uncertainties on the position angles $\left(\epsilon_{\mathrm{PA}}\right)$, and it must be corrected using $\delta \phi^{2}=\delta \phi_{\text {obs }}^{2}-\epsilon_{\mathrm{PA}}^{2}=\left(12^{\circ}\right)^{2}$ (Crutcher et al. 2004). Hence, introducing $\delta \phi=12^{\circ}$ in Eq. (7), we obtained $B_{0}=47, \mu \mathrm{G}$, which is in well agreement with the result of the full ADF analysis, given the uncertainties. We conclude that the results are robust with respect to our assumptions. 


\section{Conclusions}

We have performed polarimetric observations of the dust thermal emission at $1.4 \mathrm{THz}$ in the class 0 protostar IRAS 15398. Our data unveil a very ordered magnetic field. The direction of the magnetic field is consistent within uncertainties with that found by Franco \& Alves (2015) using optical data, which trace large cloud scales. This suggests that during its evolution the core preserved the $B$-field morphology inherited from the parental cloud, and experienced a magnetically driven collapse.

The magnetic field lines on the south-east side of the core pinch inwards, hinting at the hourglass shape predicted by the theoretical models. However, we lack the sensitivity to clearly identify the hourglass shape. The lack of such a pinching on the other side of the core may be linked to the presence of an extended structure linked to the central core. The polarisation vectors detected in this filament show a significantly different direction of the magnetic field, perhaps hinting that material is being dragged towards the central object and that the infalling gas is perturbing the magnetic field morphology. Further line observations, which allow us to infer the velocity field in the source, will help us enlighten this point.

Using the modified CF method we estimate the magnetic field strength on the plane of sky to be $B_{\text {pos }}=78 \mu \mathrm{G}$, accurate within a factor of two. This value is in the lower end of the range found on similar spatial scales performed in star forming regions for example with the JCMT telescope (see e.g. Crutcher et al. 2004; Kwon et al. 2018; Soam et al. 2018; Liu et al. 2019), where $B$-strengths of $80-5000 \mu \mathrm{G}$ have been reported.

The mass-to-flux ratio that we derive $(\lambda=0.95)$ is close to the transcritical regime $(\lambda=1)$. Usually cores are found to be supercritical (see e.g. Troland \& Crutcher 2008), in contrast with molecular clouds which are often subcritical: for instance, Franco \& Alves (2015) found $\lambda=0.027-0.057$ in Lupus I. IRAS 15398 hosts a protostellar object, which indicates that indeed gravitational collapse has happened and that, on some scales, the source must be supercritical. Our observations are likely missing the angular resolution to probe those scales, and therefore we are seeing an intermediate state due to the subscritical surrounding medium. On the other hand, the uniform-to-turbulent ratio is smaller than one $\left(B_{\mathrm{t}} / B_{0}=0.267\right)$, suggesting a strongly magnetised core. This scenario is also supported by the lack of a large Keplerian disc in the source; Yen et al. (2017) found an upper limit to the disc size of $30 \mathrm{AU}$. Magnetic braking is in fact an efficient way to remove angular momentum from the infalling and rotating material and prevent the formation of large discs ( $\mathrm{Li}$ et al. 2014).

Overall, our data suggest that IRAS 15398 evolved in a highly magnetised environment, and that the ordered magnetic field was preserved from cloud scales down to core scales. Future polarimetric observations with ALMA at high resolution will allow us to investigate whether this uniform magnetic field is preserved down to disc and envelope scales.

Acknowledgements. Based on observations made with the NASA/DLR Stratospheric Observatory for Infrared Astronomy (SOFIA). SOFIA is jointly operated by the Universities Space Research Association, Inc. (USRA), under NASA contract NNA17BF53C, and the Deutsches SOFIA Institut (DSI) under DLR contract 50 OK 0901 to the University of Stuttgart. This research has made use of data from the Herschel Gould Belt survey (HGBS) project (http:// gouldbelt-herschel.cea.fr). The HGBS is a Herschel Key Programme jointly carried out by SPIRE Specialist Astronomy Group 3 (SAG 3), scientists of several institutes in the PACS Consortium (CEA Saclay, INAF-IFSI Rome and
INAF-Arcetri, KU Leuven, MPIA Heidelberg), and scientists of the Herschel Science Center (HSC). The authors thank the anonymous referee for his/her helpful comments.

\section{References}

Allen, A., Li, Z.-Y., \& Shu, F. H. 2003, ApJ, 599, 363

Alves, F. O., Frau, P., Girart, J. M., et al. 2014, A\&A, 569, L1

Andersson, B. G., Lazarian, A., \& Vaillancourt, J. E. 2015, ARA\&A, 53, 501

Andre, P., Ward-Thompson, D., \& Barsony, M. 2000, in Protostars and Planets

IV, eds. V. Mannings, A. P. Boss, \& S. S. Russell (Tucson: University of Arizona Press), 59

André, P., Men'shchikov, A., Bontemps, S., et al. 2010, A\&A, 518, L102

André, P., Di Francesco, J., Ward-Thompson, D., et al. 2014, in Protostars and Planets VI, eds. H. Beuther, R. S. Klessen, C. P. Dullemond, \& T. Henning (Tucson: University of Arizona Press), 27

Arzoumanian, D., André, P., Didelon, P., et al. 2011, A\&A, 529, L6

Benedettini, M., Pezzuto, S., Burton, M. G., et al. 2012, MNRAS, 419, 238

Bethell, T. J., Chepurnov, A., Lazarian, A., \& Kim, J. 2007, ApJ, 663, 1055

Bjerkeli, P., Jørgensen, J. K., \& Brinch, C. 2016, A\&A, 587, A145

Bohlin, R. C., Savage, B. D., \& Drake, J. F. 1978, ApJ, 224, 132

Brauer, R., Wolf, S., \& Reissl, S. 2016, A\&A, 588, A129

Chandrasekhar, S., \& Fermi, E. 1953, ApJ, 118, 116

Chiang, H.-F., Looney, L. W., \& Tobin, J. J. 2012, ApJ, 756, 168

Coudé, S., Bastien, P., Houde, M., et al. 2019, ApJ, 877, 88

Crutcher, R. M., Nutter, D. J., Ward-Thompson, D., \& Kirk, J. M. 2004, ApJ, 600,279

Dzib, S. A., Loinard, L., Ortiz-León, G. N., Rodríguez, L. F., \& Galli, P. A. B. 2018, ApJ, 867, 151

Franco, G. A. P., \& Alves, F. O. 2015, ApJ, 807, 5

Frau, P., Girart, J. M., Zhang, Q., \& Rao, R. 2014, A\&A, 567, A116

Galli, D., \& Shu, F. H. 1993, ApJ, 417, 243

Girart, J. M., Rao, R., \& Marrone, D. P. 2006, Science, 313, 812

Harper, D. A., Runyan, M. C., Dowell, C. D., et al. 2018, J. Astron. Instrum., 7, 1840008

Hildebrand, R. H., Kirby, L., Dotson, J. L., Houde, M., \& Vaillancourt, J. E. 2009, ApJ, 696, 567

Houde, M., Vaillancourt, J. E., Hildebrand, R. H., Chitsazzadeh, S., \& Kirby, L. 2009, ApJ, 706, 1504

Hull, C. L. H., \& Zhang, Q. 2019, Front. Astron. Space Sci., 6, 3

Jones, T. J., Bagley, M., Krejny, M., Andersson, B. G., \& Bastien, P. 2015, AJ, 149,31

Jones, T. J., Gordon, M., Shenoy, D., et al. 2016, AJ, 151, 156

Jørgensen, J. K., Bourke, T. L., Myers, P. C., et al. 2007, ApJ, 659, 479

Kauffmann, J., Bertoldi, F., Bourke, T. L., Evans, N. J., I., \& Lee, C. W. 2008, A\&A, 487, 993

Kwon, W., Looney, L. W., Mundy, L. G., Chiang, H.-F., \& Kemball, A. J. 2009, ApJ, 696, 841

Kwon, J., Doi, Y., Tamura, M., et al. 2018, ApJ, 859, 4

Lazarian, A. 2007, J. Quant. Spectr. Rad. Transf., 106, 225

Li, H.-b., Fang, M., Henning, T., \& Kainulainen, J. 2013, MNRAS, 436, 3707

Li, H. B., Goodman, A., Sridharan, T. K., et al. 2014, in Protostars and Planets VI, eds. H. Beuther, R. S. Klessen, C. P. Dullemond, \& T. Henning (Tucson: University of Arizona Press), 101

Liu, J., Qiu, K., Berry, D., et al. 2019, ApJ, 877, 43

Matthews, B. C., McPhee, C. A., Fissel, L. M., \& Curran, R. L. 2009, ApJS, 182, 143

McKee, C. F., \& Ostriker, E. C. 2007, ARA\&A, 45, 565

Mouschovias, T. C. 1991 , ApJ, 373, 169

Mouschovias, T. C., \& Spitzer, L. J. 1976, ApJ, 210, 326

Nakamura, F., \& Li, Z.-Y. 2008, ApJ, 687, 354

Ostriker, E. C., Stone, J. M., \& Gammie, C. F. 2001, ApJ, 546, 980

Oya, Y., Sakai, N., Sakai, T., et al. 2014, ApJ, 795, 152

Padoan, P., Goodman, A., Draine, B. T., et al. 2001, ApJ, 559, 1005

Pelkonen, V. M., Juvela, M., \& Padoan, P. 2009, A\&A, 502, 833

Planck Collaboration Int. XXXV. 2016, A\&A, 586, A138

Rygl, K. L. J., Benedettini, M., Schisano, E., et al. 2013, A\&A, 549, L1

Santos, F. P., Ade, P. A. R., Angilè, F. E., et al. 2017, ApJ, 837, 161

Soam, A., Pattle, K., Ward-Thompson, D., et al. 2018, ApJ, 861, 65

Tachihara, K., Dobashi, K., Mizuno, A., Ogawa, H., \& Fukui, Y. 1996, PASJ, 48, 489

Troland, T. H., \& Crutcher, R. M. 2008, ApJ, 680, 457

Vaillancourt, J. E. 2006, PASP, 118, 1340

Wang, J.-W., Lai, S.-P., Eswaraiah, C., et al. 2019, ApJ, 876, 42

Whittet, D. C. B., Hough, J. H., Lazarian, A., \& Hoang, T. 2008, ApJ, 674, 304

Yen, H.-W., Koch, P. M., Takakuwa, S., et al. 2017, ApJ, 834, 178 


\section{Appendix A: Stokes parameters}

Figure A.1 reports in the three panels the Stokes parameters observed with SOFIA. The maps have been smoothed to $42^{\prime \prime}$ with respect to a native resolution of $19^{\prime \prime}$ to improve the S/N.
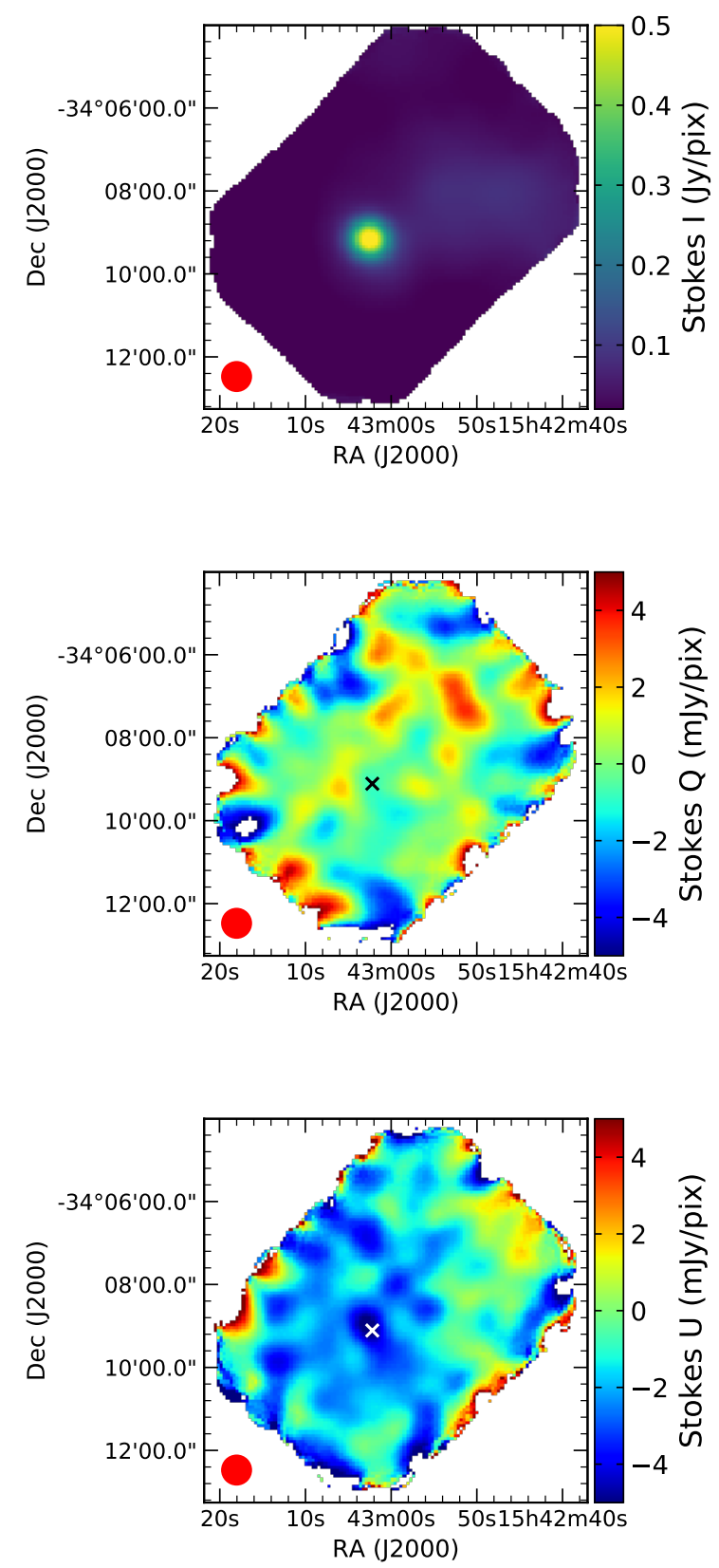

Fig. A.1. Colour map of the three Stokes parameter $I, Q$, and $U$ (from top to bottom). The smoothed beam size is indicated in the bottom right corners. In the central and bottom panels, the cross represents the position of the protostar. 